\title{
Neue Pioniere in ostalpinen Peripherräumen: die Wiederbelebung von Geisterdörfern und partiellen Wüstungen in Friaul
}

\author{
Michael Beismann ${ }^{1}$, Peter Čede $^{2}$, and Ernst Steinicke ${ }^{1}$ \\ ${ }^{1}$ Institut für Geographie, Leopold-Franzens Universität, Innsbruck, 6020, Österreich \\ ${ }^{2}$ Institut für Geographie und Raumforschung, Karl-Franzens Universität, Graz, 8010, Österreich \\ Correspondence: Michael Beismann (michael.beismann@uibk.ac.at)
}

Received: 18 December 2020 - Revised: 1 November 2021 - Accepted: 9 November 2021 - Published: 27 January 2022

Kurzfassung. This study is based on amenity migration as theoretical concept and thus on the assumption that new in-migration improves the appearance, resilience and socioeconomic situation of remote alpine regions. Current revitalisation processes in Friulian alpine villages, having become sparsely inhabited or indeed real ghost villages, are particularly suitable for such studies. The research builds on the one hand on analysis of the current state of the art as well as on analyses of official statistical data. It is on the other hand derived from own surveys in the course of investigative visits to a large part of the Friulian ghost towns. In these remote areas locals and newcomers were interviewed personally. The results show that newcomers bring innovation and new impulses in agriculture, tourism, artistic and cultural sphere and play a key role in renovating existing buildings, reusing fallow plots of land and establishing a creative dynamic. Their activities increasingly enable the regeneration and maintenance of alpine cultural landscapes, resulting in new social and agrarian structures, which are emerging as „new farming“ in the study area. The village of Dordolla in Val Aupa has recently established itself as a centre of innovation in the Friulian mountains. The influx of new pioneers and their achievements are also evident in the rest of the valley. The regeneration processes analyzed in the study area could also be transferred to other remaining partially abandoned settlements in Friuli. The motivations, solutions and ways of thinking of new pioneers combined with the increasingly positive connotations of such areas make the regeneration of the cultural landscape and potential repopulation in other isolated areas by no means unrealistic.

\section{Motivation}

Im Jahr 2007 erschien in dieser Zeitschrift ein Beitrag über die besonders hohe Konzentration völlig verlassener Siedlungen (,Ghosttowns“) in Friaul, dessen Berggebiet seit dem Zweiten Weltkrieg alpenweit die grössten Bevölkerungsverluste hinnehmen musste (Čede und Steinicke, 2007). Aus den damals publizierten Kartierungsergebnissen war die mittlerweile relevant gewordene New Highlander- bzw. Amenity Migration ${ }^{1}$ noch nicht ableitbar. Seit rund 40 Jahren ist in

\footnotetext{
${ }^{1}$ Um die neuen Gebirgsbewohner zu definieren, wurde eine Reihe von Begriffen geschaffen, für die es im Deutschen kaum oder nur holprige Übersetzungen gibt, wie z. B. ,amenity (led) migrants“, „multi-local residents“, ,lifestyle migrants“ oder eben ,,new high-
}

Teilen der Alpen eine demographische Trendumkehr feststellbar: Menschen aus ausseralpinen Räumen finden in zunehmendem Masse Natur und Kultur abgelegener Gebirgsregionen so attraktiv, dass sie ihren permanenten, saisonalen oder episodischen Wohnsitz dorthin verlegen. Dieser neue Mobilitätstypus, der inzwischen mehrfach belegt und auch quantifiziert wurde (zuletzt Steinicke und Löffler, 2019), hat in den 2000er Jahren auch die ersten Berggemeinden Nordfriauls (Montagna Friulana) erfasst. Der verstärkte Zuzug ins

landers“ (Moss, 2006; Löffler und Steinicke, 2007; Bender und Kanitscheider, 2012; McIntyre, 2009; Gosnell und Abrams, 2011). Zudem wird - v. a. in Schweizer Publikationen - für die multilokale Bevölkerung auch der Begriff ,Zweitheimische“ verwendet (vgl. zuletzt Zimmerli, 2021). 
Gebirge ist aktuell in den ehemaligen friulanischen „totalen“ und ,partiellen“ Ortswüstungen besonders deutlich. So liessen sich bereits 2010 und 2012 nur mehr wenige echte Geisterdörfer nachweisen (Steinicke et al., 2012, 2014). Während im friulanischen Berggebiet - trotz der neuen Gebirgsbewohner $^{2}$ - insgesamt noch immer ein negativer Wanderungssaldo vorherrscht, sind in den bis in die 2000er Jahre entsiedelten und entsiedlungsgefährdeten Ortschaften im Canale di Ferro und seinen Seitentälern, von wenigen Ausnahmen abgesehen, Zuwanderungen registrierbar.

Diese Siedlungen bieten somit gleichsam Laborbedingungen, um physiognomische, funktionale, soziale und wirtschaftliche Auswirkungen der neuen Gebirgsbewohner aufzuzeigen. Dabei lässt sich eine Reihe von Innovationen beobachten, die möglicherweise auf die gesamte Montagna Friulana sowie auf andere alpine Periphergebiete übertragbar sind. Es scheint daher berechtigt, die Mehrheit der Neuzuwanderer als ,neue Pioniere“ (new pioneers; Jacob, 1997) zu bezeichnen. Aufgabe vorliegender Studie soll es somit sein, ihre raumwirksamen Handlungen darzulegen.

Bereits ein kurzer Besuch in den aktuell nur mehr vereinzelt durch fortschreitenden Verfall gekennzeichneten Ortschaften zeugt von Revitalisierungen des Baubestandes und agrarischen Aktivitäten der Zuwanderer. Es gilt in den folgenden Abschnitten festzustellen, ob es überhaupt noch Geisterdörfer in Friaul gibt und welche Innovationen die Zuwanderer bewirken. Handelt es sich lediglich um Wiederaufnahme der aufgegebenen landwirtschaftlichen Tätigkeit? Ist eine neue Landnutzung zu beobachten? Folgt man postproduktivistischen Ideen (Wilson, 2009), bei denen ökonomische Profite zugunsten von Selbstversorgung, biologischem Landbau, Lifestyle oder kulturlandschaftlicher Revitalisierung in den Hintergrund treten? Ein weiteres Augenmerk ist auf die Frage zu richten, ob die Aktivitäten der neuen Gebirgsbewohner anderen, ausseragrarischen Aktionsfeldern Impulse verleihen können: Die Rekultivierung landwirtschaftlicher Nutzflächen stärkt zweifellos ,,ländliche Attraktivitätswerte“ (Meyer, 1998:161-164) und mindert Naturrisiken im Gebirge. Gleichzeitig ist der Gedanke naheliegend, dass diese Art von multifunktionaler Landwirtschaft die notwendige scenic dimension (Vejre et al., 2007; Dissart und Marcouiller, 2012) als Voraussetzung bietet, um auch in anderen Bereichen innovativ auf Dorf- und Regionalentwicklung zu wirken.

\section{Grundkonzeption und Forschungsdesign}

Um die neue Zuwanderung in Peripherräume - vor allem ins Gebirge - und die damit einhergehende Verschiebung der Wohnsitzpräferenz vom urbanen Raum in abgelegene, aber als attraktiv wahrgenommene ländliche Regionen zu erklä-

\footnotetext{
${ }^{2}$ Um den Text nicht zu überladen, gelten sämtliche in dieser Arbeit verwendeten personenbezogenen Bezeichnungen für alle $\mathrm{Ge}$ schlechter.
}

ren, bieten sich als theoretischer Rahmen dieses Beitrags die Konzepte der Counterurbanisierung (Berry, 1976) und Amenity Migration (Graves, 1980; Moss, 2006) an.

Im Unterschied zur Counterurbanisierung umfasst die Amenity Migration auch die multilokal lebende Bevölkerung (McIntyre, 2009; Duchêne-Lacroix et al., 2018) und bildet dementsprechend einen Mobilitätstypus, der nicht unbedingt mit einer permanenten Wohnsitzverlagerung einhergeht. Dieses Phänomen ist auch die treibende Kraft der gegenwärtigen Wiederbesiedelung und des aktuellen Bevölkerungszuwachses in vielen Teilen des Alpenraums. Die Hauptmotive der Amenity Migranten für einen Umzug ins Gebirge sind die naturräumlichen Vorzüge, eine hohe Lebensqualität, das unterschiedliche Freizeitangebot, ein günstiger Wohnungsmarkt sowie die räumliche Unabhängigkeit vom Arbeitsplatz aufgrund der modernen Kommunikationstechniken. Dieser Typus von freiwilligen Wanderungen, über dessen Spezifika in den Alpen Perlik im Jahr 2006 erstmals publiziert hat, ist bereits mehrfach Gegenstand von grundlegenden Überblicksdarstellungen gewesen (vgl. Moss und Glorioso, 2014, Kordel et al., 2018, oder zuletzt Steinicke und Löffler, 2019).

Neuere alpenbezogene Arbeiten zu dieser Thematik befassen sich auch mit New Farming (z. B. Gretter et al., 2019), auf das noch später zurückzukommen sein wird, sowie mit Verbesserungen der Lebensqualität und Veränderungen des Lifestyles durch Zuwanderung in Gebirgsräume (z. B. Cretton, 2019). Ähnlich wie Perlik (2011) verweist die zuletzt genannte Autorin auf eine daraus resultierende Gentrifizierung in Teilen der Alpen, insbesondere in Tourismusregionen. Zäch et al. (2015) bringen wiederum die neuen Gebirgsbewohner mit landschaftlicher Attraktivität in Verbindung und fassen solche Räume unter dem Begriff Amenity Landscapes zusammen. In Regionen, wo diese nicht oder nur unzureichend wahrgenommen wird, kommt es hingegen zu keiner nennenswerten Zuwanderung von Amenity Migranten (Čede et al., 2018).

In aktuellen Studien zum Forschungsschwerpunkt über neue Gebirgsbewohner wird zudem nicht nur auf freiwillige Zuwanderungen in Berggebiete eingegangen. Einige Autoren beziehen nunmehr auch die durch Gewalt oder Constraints veranlasste unfreiwillige Migration ein. Zum Beispiel stellt Graf (2020) Arbeitsmigranten in Graubünden in den Vordergrund ihrer Untersuchung. Ein kürzlich von Perlik et al. (2019) herausgegebener Sammelband befasst sich mit der Thematik von Flüchtlingen als neue Gebirgsbewohner. In einigen Studien werden Projekte zur Unterstützung benachteiligter Gruppen - insbesondere Asylsuchende - durch Arbeitsmöglichkeiten als ,soziale Innovation“ betrachtet (Dematteis et al., 2018, Fourny, 2018 oder Perlik und Membretti, 2018). Wie Tschumi et al. (2020) ausführlich diskutieren, sind ,soziale Innovationen“ im wissenschaftlichen Schrifttum allerdings unterschiedlich definiert. In Anlehnung an Ayob et al. (2016) erscheint es zweckmäßig, sie als eine Form der Zusammenarbeit zu sehen, die zu neuen Ideen und deren 
Umsetzung führt. Im Kontext der Regionalentwicklung ist „neu“ als ,,aussergewöhnlich“ für das Untersuchungsgebiet $\mathrm{zu}$ verstehen.

Vorliegende Studie, die sich mit Innovationen in strukturschwachen alpinen Regionen auseinandersetzt, fokussiert auf freiwillige Amenity Migration. Dabei konzentrieren sich die Autoren auf jene Innovationen, die von neuen Zuwanderern ausgehen und vor Ort räumlich wirksam sind. Insofern werden diese hier synonym als Pionierleistungen bezeichnet. Letztere betreffen einerseits Impulse durch Projekte und Aktivitäten, die im Untersuchungsgebiet neu sind, wie z. B. die Durchführung von Konzerten oder Ausstellungen, andererseits - v. a. im Agrarsektor - die Wiederbelebung von aufgegebenen landwirtschaftlichen Strukturen. Ähnlich wie bei Viazzo und Zanini (2014) über die Kreativität von Neuzuwanderern oder bei Mayer und Meili (2016) über die Bedeutung von Amenity Migranten für Unternehmensgründungen sind Innovationen im gegenständlichen Beitrag nicht im zeit-räumlichen Sinn als Ausbreitungsprozess (Hägerstrand, 1969) aufzufassen. Dieser würde eine zeitliche Längsschnittanalyse erfordern, die hier nicht vorgesehen ist.

Die vorhin diskutierten Pionierleistungen lassen sich sehr gut in ehemaligen Geisterdörfern sowie in partiellen Wüstungen im Zuge von Wiederbesiedelung und Revitalisierung aufzeigen. Hinsichtlich der Neubewertung solcher marginalisierter Siedlungen liegt insofern eine Lücke im aktuellen Forschungsstand vor. Ziel der Arbeit ist es daher, Zahl, Herkunft und Struktur der neuen Pioniere in den vormaligen friulanischen Geisterdörfern und partiellen Wüstungen sowie die damit im Zusammenhang stehenden Innovationen in der Kulturlandschaft und Wirtschaft zu analysieren. Dem Beitrag liegen folgende Annahmen zugrunde:

- Mit der Anwesenheit neuer Pioniere geht sowohl eine Sanierung von Wohngebäuden als auch eine Revitalisierung brachgefallener Fluren einher.

- Dies führt mehrfach zu neuen agrarischen Strukturen im Gebirge und damit wiederum zur Bildung einer neuen sozialen Kategorie: jener der New Farmer.

- Zudem spiegeln sich die Aktivitäten der Pioniere in anderen innovativen Aktionsfeldern im sozioökonomischen und kulturellen Bereich wider.

Die Studie stützt sich auf einen Methodenpool, der multiperspektivische Zugänge vereint (Schnell et al., 2018). Das Forschungsdesign sieht dabei zwei Arbeitsschritte vor: eine generelle Übersicht über die gegenwärtige Situation in den ehemaligen Geisterdörfern und partiellen Wüstungen des Canale di Ferro sowie eine detaillierte Untersuchung in der Gemeinde Moggio Udinese. Neben der Berücksichtigung des aktuellen Forschungsstandes und der Auswertung amtlicher Statistiken war zunächst die Aktualisierung von Nutzungs- bzw. Funktionskartierungen in den bis in die 2000er Jahre durch Entvölkerung gekennzeichneten Siedlungen notwendig. Dabei wurden im Zeitraum von 2018 bis 2020 alle im Jahr 2005 kartierten Geisterdörfer (vgl. Kap. 1) nochmals aufgesucht und die aktuelle Bewohnerzahl eruiert sowie die Gebäudefunktion und Landnutzung kartiert. $\mathrm{Zu}$ den wichtigsten Erhebungsmethoden vor Ort zählten unter anderem teilstrukturierte Interviews mit allen während der Feldforschungen anwesenden Bewohnern, wobei Herkunft, Anwesenheitsdauer, Beruf, Besitzverhältnisse und Alter der Zuwanderer im Mittelpunkt standen.

Die tiefer gehenden Vor-Ort-Erhebungen in den ehemaligen Geisterdörfern und partiellen Wüstungen in der Beispielsgemeinde Moggio Udinese wurden durch die Mitarbeit von 34 Studierenden der Universitäten Innsbruck, Graz und Triest erleichtert, die im Herbst 2018 und Frühjahr 2019 ihre Abschlussmodule (Mastermodule) in den strukturschwachen Berggebieten Friauls absolvierten. Dadurch war es möglich, dort alle Haushalte aufzusuchen und die Anwesenden zu interviewen. Von den insgesamt 151 Haushalten in den nordfriulanischen Geisterdörfern (Kap. 4) fielen 111 auf die Gemeinde Moggio Udinese. Die Interviews umfassten sowohl permanente als auch zugewanderte, multilokale Bewohner. Basis der Datenakquirierung waren - wie dargelegt - teilstrukturierte Interviews, die in italienischer Sprache geführt wurden. Obwohl die Befragten über den wissenschaftlichen Hintergrund der Untersuchung informiert waren, zeigte ein Grossteil Misstrauen gegenüber der Verwendung audiovisueller Aufnahmetechniken, mitunter auch gegenüber schriftlichen Notizen. Diese Haltung lässt sich insofern erklären, dass es sich bei den Neubewohnern vorwiegend um eine multilokal lebende Bevölkerung handelt, bei der aus Gründen der Steuerersparnis nicht selten ein Familienmitglied den Hauptwohnsitz im Gebirge gemeldet hat ${ }^{3}$. Da diese Personen Aufzeichnungen über ihre Lebensverhältnisse ablehnten, wurden Gedächtnisprotokolle erstellt. Dabei ist nicht ausser Acht zu lassen, dass in den kleinen Ortschaften haushaltsbezogene Aussagen leicht zu verorten und damit anonyme Auswertungen kaum möglich sind. Da qualitative Inhaltsanalysen im Sinne von Mayring (2019) mit Kategorisierungen nicht möglich waren, bezog sich die Datenanalyse auf summarische Zusammenfassungen. Die Erhebungen in der Gemeinde Moggio Udinese gingen über die oben angeführten Fragestellungen hinaus und umfassten zusätzliche Interviewpunkte über die landwirtschaftlichen, aber auch über die ausseragrarischen Tätigkeiten in den ehemaligen Geisterdörfern und partiellen Wüstungen sowie über die Beziehungen zwischen Einheimischen und Zugewanderten. Weitere Hinweise über die spezifischen Arbeitstechniken sind in den entsprechenden Abschnitten angeführt. Seit der intensiven Feldforschungsphase betreiben die Autoren ein ständiges Monitoring mit wiederholten Geländeaufenthalten sowie mit SocialMedia-Kontakten, die auch während der Covid-19-Situation im Jahr 2020 und 2021 weitergeführt wurden.

\footnotetext{
${ }^{3}$ Man spart sich somit die Zweitwohnsitzabgabe (IMU - Imposta municipale propria)
} 
Es erscheint zweckmässig, zunächst beispielhaft Pionierleistungen im gesamten Alpenraum aufzuzeigen und dann auf die demographische Sonderstellung des friulanischen Gebirges einzugehen. Ein eigener Abschnitt widmet sich der Neubesiedlung nordfriulanischer Geisterdörfer, ehe die Comune Moggio Udinese als Fallbeispiel für konkrete Revitalisierungsmassnahmen in Entvölkerungsgebieten vorgestellt wird. Bedeutung und Transfermöglichkeiten sowie der Mehrwert der Revitalisierung von Ortswüstungen bilden die Basis für die abschliessende Diskussion.

\section{Innovationen durch Pionierleistungen im Alpenraum}

Obwohl die in der Fachliteratur ausführlich dargelegte Amenity Migration (Moss, 2006; Moss und Glorioso, 2014) kein Massenphänomen ist, wurde sie im Alpenraum seit den 1990er Jahren ein relevanter Faktor für die Aufwertung peripher liegender Orte (Perlik, 2006). Selbst wenn die Wiederbesiedlung in Personenzahlen gemessen nicht überall statistisch gleich relevant ist, so sind oft schon wenige Zuwanderer in der Lage, als Synergieeffekt ihrer Lebensgestaltung eine Neubelebung zu initiieren. Forschungsergebnisse aus den Westalpen (Löffler et al., 2016), wo die Zuwanderung in demographische Problemgemeinden früher begonnen hat als in den Ostalpen, zeigen, dass durch diese positiven Impulse zudem die Überalterung ebenso abgeschwächt wird wie der negative Geburtensaldo.

Die Thematik, ,agrarische Innovationen durch Neuzuwanderer" in alpinen Periphergebieten wurde bereits mehrfach aufgegriffen: Hier ist zunächst das Phänomen des „néoruralisme“ zu nennen, das zu Beginn der 1970er Jahre im Süden der französischen Alpen erstmals in Erscheinung trat (Garcia, 1977; Mercier und Simona, 1983). Junge, ausseralpine Zuwanderer haben sich dort niedergelassen und sowohl die jahrzehntelangen Bevölkerungsverluste ausgeglichen als auch durch ihre agrarischen Aktivitäten im Laufe der Jahre die Kulturlandschaft revitalisiert. Mit den neuen landwirtschaftlichen Tätigkeiten verbanden sich zudem überregionale touristische Impulse (Warmuth et al., 2016). In den benachbarten piemontesischen Alpen, wo sich ab den 1980er Jahren ebenfalls neue Bewohner ansiedelten, beobachteten Fassio et al. (2014) eine Wiederbelebung von Familienbetrieben im Agrarbereich - ähnlich wie Varotto und Lodatti (2014) in den venezianischen Alpen. Jüngst haben sich auch Gretter et al. (2019) diesem Thema zugewandt und New Farming als soziale Innovation im Sinne der eingangs erwähnten postproduktivistischen Bedingungen erkannt.

Die Innsbrucker Arbeitsgruppe DCA (Demographic Change of the Alps) hat sich in ihren Untersuchungen darüber hinaus mit Pionierleistungen alpiner Newcomer in anderen Bereichen befasst ${ }^{4}$. Dabei fallen die kulturellen In-

\footnotetext{
${ }^{4}$ https://www.uibk.ac.at/geographie/migration/am/pub.html (letzter Zugriff: 12. Oktober 2021).
}

novationen in Gebieten mit sprachlichen Minderheiten v. a. bei den Okzitanen und Walsern im Piemont und im Aostatal sowie bei den Sprachinseldeutschen in Friaul auf. Obwohl mehrheitlich der italienischen Sprache zugehörig, haben die neuen Bewohner Veranstaltungen der Minderheiten (mit-)organisiert und dabei wertvolle Impulse erzeugt, die kulturelle Vielfalt zu bewahren (z. B. Steinicke et al., 2011). Im aostanischen Gressoney-la-Trinité unter dem Monte-Rosa-Massiv, im dolomitenladinischen Fassatal sowie in der höchstgelegenen friulanischen Gemeinde Sauris/Zahre gehörten Touristen aus den Ballungszentren Oberitaliens zu den ersten, die ihre Freizeitwohnsitze im Gebirge zu saisonal genutzten weiteren Wohnsitzen umgewandelt haben. Dabei gab man den aus rustikalen Städeln und Hütten umgebauten Appartments nicht selten deutsche Namen - wie „Edelweiss“ oder „Heidi“.

Schon vor einigen Jahren analysierten Mayer und Meili (2016) in den Schweizer Alpen den innovativen Beitrag von Unternehmungsgründungen durch Neuzuwanderer, der trotz infrastruktureller Schwierigkeiten regionalwirtschaftliche Impulse setzen konnte. Andere Pionierleistungen erkannten Grüner und Konzett (2017) in peripher gelegenen Siedlungen Osttirols: Neuzuwanderer im Tiroler Gailtal, wo seit den 1970er Jahren Bevölkerungsrückgänge zu verzeichnen sind, haben dort ausschlaggebende Impulse für die Entwicklung des Biathlon-Sports im Bezirk Lienz geschaffen. Ausserdem traten sie durch Erwerb und Revitalisierung leerstehender Bauernhöfe und landwirtschaftlicher Nutzflächen hervor. Damit zeigten sie, inwieweit neue Gebirgsbewohner in der Lage sind, das aktuell grösste Problem der Tiroler Berglandwirtschaft, nämlich die Frage nach der künftigen Hofübernahme, zu verkleinern.

Die bisher geschilderten Beispiele betrafen Pionierleistungen durch Zuwanderer in alpine Peripherräume, nicht jedoch in Geisterdörfer. In diesem Zusammenhang sei vorerst auf einen Sonderfall im äussersten Westen Friauls zu verweisen. Die Ortschaften Erto und Casso (1961 zusammen 1961 Ew.) wurden von der Staudammkatastrophe von Vajont (Longarone) im Jahr 1963 so stark erfasst, dass der italienische Staat grosse Teile der Überlebenden aus Gründen der Sicherheit zur Umsiedlung zwang (Steinicke, 1991:142-155). Das Dorf Casso wurde damit zur grössten Katastrophen-Wüstung der Alpen. Seit den 1990er Jahren entwickelte sich die Gegend um den ehemaligen Vajont-Stausee jedoch zu einem Zuwanderungsgebiet (2019: 1372 Ew.). In ihren Untersuchungen fanden Beismann et al. (2015) heraus, dass die Neuzuwanderer zahlreiche Innovationen im kulturellen und gastronomischen Bereich, v. a. aber in privaten Bauaktivitäten sowie im Tourismus schufen. Bemerkenswert ist dabei, dass die Revitalisierung von Bewohnern von Zweitwohnsitzen initiiert wurde, worauf zunächst Rückwanderer folgten. Von der geförderten Albergo Diffuso-Bewegung beschleunigt, entwickelten sich Erto und Casso in kurzer Zeit zu lebensfähigen Ortschaften, was weitere Rückwanderer, neue Zuwanderer 
und einen längeren, aussertouristischen Aufenthalt der Besitzer von Zweitwohnsitzen zur Folge hatte.

\section{Die Montagna Friulana als demographischer Sonderfall}

Alle Berggemeinden Friauls sind seit Generationen von einer massiven Abwanderung geprägt (Zanini, 1964). Bis in die 1950er Jahre konnte der Geburtenüberschuss die Entvölkerungsraten noch einigermassen auffangen, danach machten sich die biodemographischen Auswirkungen der vorangegangenen Abwanderungsphasen bemerkbar. Die Erdbebenkatastrophen von 1976, die den nordfriulanischen Siedlungsraum weitflächig zerstört hatten, zogen als Folge eine Konzentration der Bevölkerung in den Gemeindehauptorten und verstärkte Einwohnerverluste in der Peripherie nach sich. Dies beschleunigte die Entstehung von Orts- und Flurwüstungen und damit den Verfall der Kulturlandschaft. Während die Kartierung von Steinicke (1991) undifferenziert die aufgelassenen und wüstungsgefährdeten Siedlungen Friauls am Ende der 1980er Jahre zeigt, wurde in der eingangs bereits angesprochenen Kartierung von Čede und Steinicke (2007) zwischen völlig entsiedelten und zeitweise bewohnten Ortschaften unterschieden. Die Karte zeigt sämtliche Ortswüstungen im Canale di Ferro, d. i. der untere Abschnitt des Fellalaufs, sowie in dessen Seitentälern (Abb. 1). Dieser von der Entvölkerung am schwersten betroffene nordöstliche Teil Friauls bildet den Untersuchungsraum vorliegender Studie.

Auch heute zeigt der statistische Befund der Montagna Friulana weiterhin Bevölkerungsverluste, jedoch ist unübersehbar, dass sie geringer werden (Tabelle 1) und die gegenwärtige demographische Entwicklung nicht von jener anderer italienischer Berggebiete abweicht (Löffler et al., 2016). Zwar ist die Zahl der Zuwanderer geringer als in den lombardischen oder piemontesischen Alpen, doch trifft man mittlerweile in allen Talschaften Friauls - selbst in äusserst abgelegenen Teilen - auf neue Bewohner oft ausseralpiner Herkunft. Dass dieser Prozess hier im Osten später einsetzte und langsamer verläuft, ist zum einen auf die vorhin angesprochenen ungünstigen bevölkerungsbiologischen Strukturen zurückzuführen. Zum anderen fehlen - im Unterschied zu den westlichen und mittleren italienischen Alpen - grosse Ballungsräume, wie Turin, Mailand, Brescia oder Verona, die als Quellgebiete für Zuwanderung in benachbarte Bergregionen besonders relevant sind. Insgesamt hat sich die Einwohnerzahl der Montagna Friulana von 132827 im Jahr 1951 bis heute halbiert (2019: 66102 Ew.), im Canale di Ferro ist sie sogar um zwei Drittel gesunken - von 15107 auf 5096 Einwohner.

Nicht alle neuen Zuwanderer im Gebirge sind ständige Bewohner. Die meisten zählen zu einer multilokalen Bevölkerung (McIntyre, 2009) mit einem ihrer Wohnsitze in der Montagna Friulana. Diese Wohnstandorte unterscheiden sich jedoch von touristischen Freizeitwohnsitzen durch längere
Tabelle 1. Bevölkerungsverluste im Berggebiet Friauls 1952-2019 (eigene Berechnungen nach ISTAT, 1951 ff.; http://www.demo.istat. it, letzter Zugriff: 1. Dezember 2022).

\begin{tabular}{lc}
\hline Zeitraum & Bevölkerungsverluste p. a. (\%) \\
\hline $1952-1961$ & 1,05 \\
$1962-1971$ & 2,29 \\
$1972-1981$ & 1,09 \\
$1982-1991$ & 0,97 \\
$1992-2001$ & 0,72 \\
$2002-2011$ & 0,20 \\
$2012-2019$ & 0,16
\end{tabular}

Aufenthaltsdauer ihrer Bewohner, die dort nicht nur urlauben bzw. konsumptiv leben, sondern oft auch im Remote Office arbeiten und am dörflichen Leben teilnehmen. Für diesen Personenkreis hat sich seit der von Moss (2006) herausgegebenen Sammelpublikation - auch in deutscher Sprache - der Begriff der Amenity Migranten durchgesetzt.

\section{Die Geisterdörfer und partiellen Wüstungen mit ihren neuen Bewohnern}

Der Vergleich von Abb. 1 und 2 zeigt das fast völlige Verschwinden totaler Ortswüstungen: Bestanden im Jahr 2005 neben den zahlreichen partiellen Ortswüstungen weitere 18 völlig verlassene Ortschaften in den Gemeinden des Canale di Ferro, so waren es im Jahr 2020 nur mehr zwei. Sowohl bei Chiout degli Uomini (1921: 68 Ew.) als auch bei Vidali di Chiusaforte (1921: 84 Ew.) handelt es sich um Ruinendörfer, die nach der Erdbebenserie von 1976 nicht mehr wiederaufgebaut wurden und bis heute völlig verfallen sind. Während Chiout degli Uomini im Raccolanatal zu Fuss nur mehr mühsam auf zugewachsenen Wegen erreichbar ist, liegt Vidali direkt an der Staatsstrasse am Talrand; der Baubestand ist dort in den letzten Jahren - aus Gründen der Sicherheit weitgehend abgetragen worden. Die Bandbreite der Wiederbesiedlung der übrigen 16 Ortschaften reicht von zwei sporadischen Bewohnern (Riulade di sotto und sopra) bis 32 multilokal wohnenden Zuwanderern Stavoli (Abb. 3).

Allerdings lässt sich bei den wenigsten die genaue Einwohnerzahl feststellen, da sie selten ständig, sondern zeitweise bewohnt und nicht immer alle Haushaltmitglieder anwesend sind. Zudem weist die amtliche Statistik diesen multilokal lebenden Bevölkerungsteil nicht aus. Aus Gründen der Steuerersparnis meldet man den weiteren Wohnsitz im Gebirge oftmals als „Hauptwohnsitz“ eines im Haushalt lebenden Partners an. Die Meldedaten bieten somit nur Anhaltspunkte, für verlässliche Aussagen ist wiederholte Feldarbeit erforderlich.

Von 151 identifizierten Haushalten in den beforschten ehemaligen Geisterdörfern und partiellen Wüstungen (Abb. 2) konnten 144 in die Erhebungen einbezogen werden. Die An- 


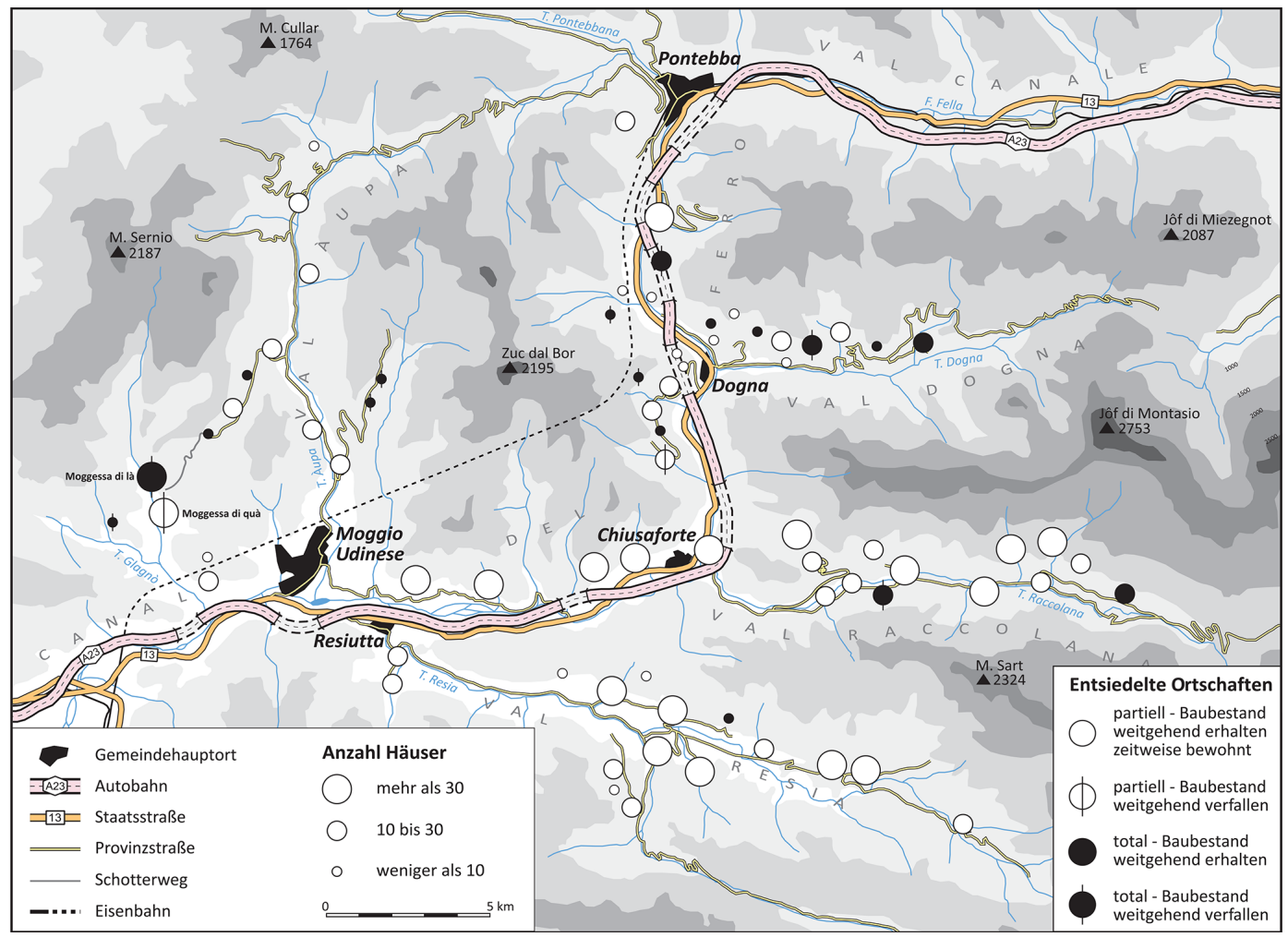

Abb. 1. Geisterdörfer und partielle Wüstungen in Nordfriaul 2005 (modifiziert nach Čede und Steinicke, 2007:98).

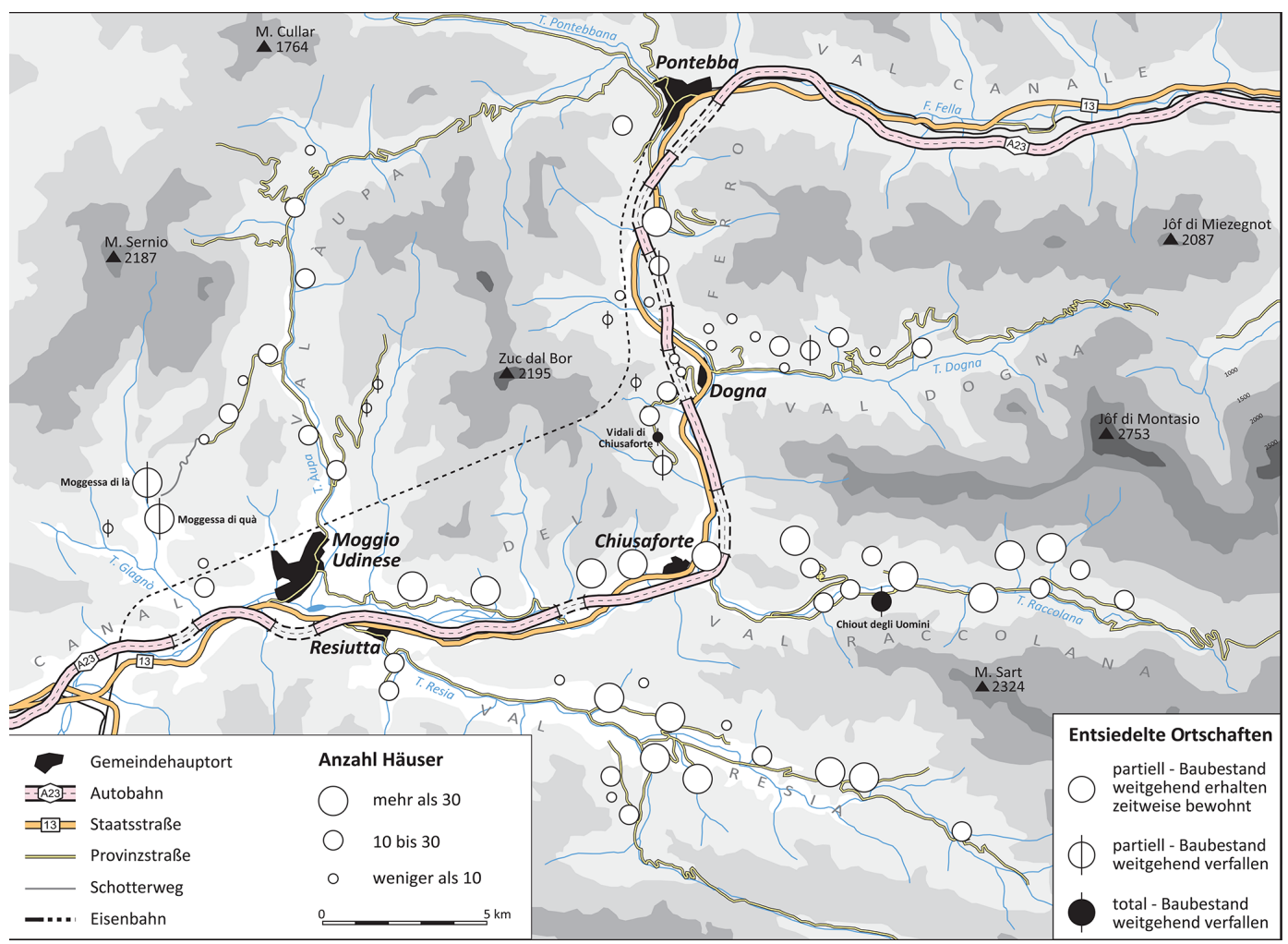

Abb. 2. Die Neubesiedlung der Geisterdörfer und partiellen Wüstungen in Nordfriaul 2020 (eigene Erhebungen). 
wesenheitsdauer der Neuzuwanderer reicht von episodischen und periodischen monatlichen Besuchen bis zu saisonalen Aufenthalten, wobei Stretti im Raccolanatal, Martignilas im Resiatal sowie Chiout Martin im Haupttal des Canale di Ferro sogar in den Wintermonaten, also ständig bewohnt sind. Im Untersuchungszeitraum 2018-2020 konnten bis auf sieben Haushalte alle in die Befragungen einbezogen werden. Auffällig ist in diesem Zusammenhang, dass die Mehrheit der Zuwanderer aus Norditalien im Jahr 2020 bereits Anfang März - am Beginn der Corona-Krise - die ehemaligen Geisterdörfer aufsuchte und dort bis Anfang Juni sowie über den Sommer hindurch blieb. Offensichtlich lässt sich dieser unüblich frühe Aufenthaltsbeginn, wo so manche Zufahrt noch von Schneeresten behindert wurde, im Sinne einer Health Migration deuten. Schulschliessungen in Italien ermöglichten in dieser Phase auch Kindern eine längere Anwesenheit in den abgelegenen Dörfern.

Die Herkunft neuer Gebirgsbewohner ist regional sehr unterschiedlich. Zahlreiche kommen als Rückwanderer der zweiten und dritten Generation aus den nahe liegenden zentraleren Ortschaften, in die ihre Eltern und Grosseltern übersiedelt waren. Eine quantitative Wiedergabe bzw. räumliche Muster von Quell- und Zielgebieten scheint jedoch nicht möglich: Inländische sowie ausländische Zuwanderer leben häufig mit einer oder mehreren Personen mit friulanischen Wurzeln im gemeinsamen Haushalt. Die Neusiedler kommen zum überwiegenden Teil aus den Städten der Region Friaul-Julisch Venetien oder aus den Gemeindehauptorten des Canale di Ferro, ferner aus den urbanen Zentren der Padania, aber ebenso aus Frankreich, Österreich, Deutschland, Grossbritannien, Schweden und der Schweiz. Selbst in ständig bewohnten Siedlungen wie Dordolla handelt es sich häufig um eine internationale, multilokal lebende Bevölkerung.

In den untersuchten 144 Haushalten war die Zahl der Pensionisten gering: Sie betrug weniger als 30 Personen. Da aber immer wieder nicht zum Haushalt gehörende ältere Personen für längere Zeit auf Besuch waren, ist auch hier die genaue Zahl schwer zu eruieren. Der überwiegende Teil der Zuwanderer ist im Erwerbsalter und - abgesehen von wenigen Ausnahmen - online mit ihren Arbeitsplätzen in den Zentralräumen Norditaliens verbunden, wo sich meist die „Hauptwohnsitze“ befinden. Auch von den saisonal anwesenden Bewohnern - v. a. den Inländern - wird der Arbeitsort zwei- bis fünfmal im Monat für einige Tage aufgesucht. Aus dem Ausland sowie aus Mittel- und Süditalien kommende Zuwanderer sind zu über zwei Drittel freiberuflich tätig (Arzt, Schriftsteller, Kunsthandwerker, Gastronom, Regisseur, Übersetzerin, Käseerzeuger u. a.). Fast alle betreiben jedoch zusätzlich noch landwirtschaftliche Tätigkeiten bzw. Gartenbau und Schaf- oder Ziegenhaltung, die auch über die Besitzgrenzen hinausreichen können. Die Nähe zur Natur, der günstige Erwerb des weiteren Wohnsitzes, die Ruhe in den abgelegenen Ortschaften, die verschiedenen Freizeitmöglichkeiten sowie die Verfügbarkeit moderner Kom- munikationstechnologien vor Ort waren die immer wieder genannten Gründe für den Zuzug.

\section{Revitalisierung peripherer Siedlungen am Beispiel der Gemeinde Moggio Udinese}

Am Schuttkegel der Aupa bei ihrer Mündung ins Haupttal der Fella (Canale di Ferro) gelegen, sind dem Gemeindehauptort Moggio Udinese das gesamte Val Aupa sowie die Dörfer des Monticello, das Paralleltal Val Alba und das Einzugsgebiet des Torrente Glagnò eingegliedert. Dort befinden sich sechs ehemalige Geisterdörfer und weitere Siedlungen unterschiedlicher Stadien von Degradation und anschliessender Wiederbelebung. Moggio Udinese selbst sowie die nahe gelegenen Fraktionen Pradis und Chiandra orientierten sich am Tal des Canale di Ferro, wo Staatsstrasse und Autobahn verlaufen. Im Jahr 2020 umfasst die Einwohnerzahl der gesamten Comune Moggio Udinese 1676 Personen.

\subsection{Bevölkerungsentwicklung und neue Bewohner}

Während der Gemeindehauptort Moggio Udinese laut kommunalem Meldebüro (ufficio anagrafico) seit der Jahrtausendwende $15 \%$ seiner Gesamtbevölkerung verloren hat, beträgt der Bevölkerungsverlust in den übrigen 24 Orten (nach der letzten Gemeindestrukturreform in neun Fraktionen zusammengefasst) $36 \%$. Allerdings zeigen die Untersuchungen in der grössten Fraktion Dordolla (Abb. 4 und5), dass über ein Viertel der Gemeldeten bereits vor 20 Jahren abgesiedelt war, den „Hauptwohnsitz“ jedoch in ihrem ehemaligen Wohnort belassen hat. Somit wird verständlich, warum die Sterbeziffer mit den Zahlen der tatsächlich anwesenden Bevölkerung nicht korrelieren, womit die Meldedaten in Bezug auf den Einwohnerstand der beforschten Siedlungen unzureichend, wenn nicht irreführend sind.

Nachvollziehbarer gestalten sich dagegen die Zahlen zur Migration. Der ausgeglichenen Wanderungsbilanz des Gemeindehauptortes steht im „Hinterland“ zwischen 1998 und 2017 eine positive Bilanz von 58 Neuanmeldungen und nur sechs Abmeldungen gegenüber. Damit sind die peripheren Fraktionen - trotz teilweiser Bevölkerungsabnahme - offiziell auch ein Zuzugsgebiet. Dazu kommen die nicht gemeldeten, saisonal anwesenden und multilokalen Bewohner, die gemeinsam oft die Mehrheit der tatsächlich Anwesenden darstellen. Da die zuletzt genannten Newcomer Aufschwung und Entwicklung in den 24 Fraktionen weitgehend bestimmen, sind gerade sie bei quantitativen Erhebungen nicht ausser Acht zu lassen. Dies kann am Beispiel des Dorfes Dordolla dargestellt werden: Allein im Jahr 2018 waren dort mindestens fünf kürzlich zugewanderte und ganzjährig anwesende Bewohner aus unterschiedlichen Gründen nicht gemeldet, obwohl sie vor Ort sogar Immobilien besassen und $15 \%$ der tatsächlich ganzjährig Anwesenden ausmachten.

Nachfolgend soll versucht werden, die Entwicklung in Dordolla mit seinen unterschiedlichen Akteursgruppen zu 


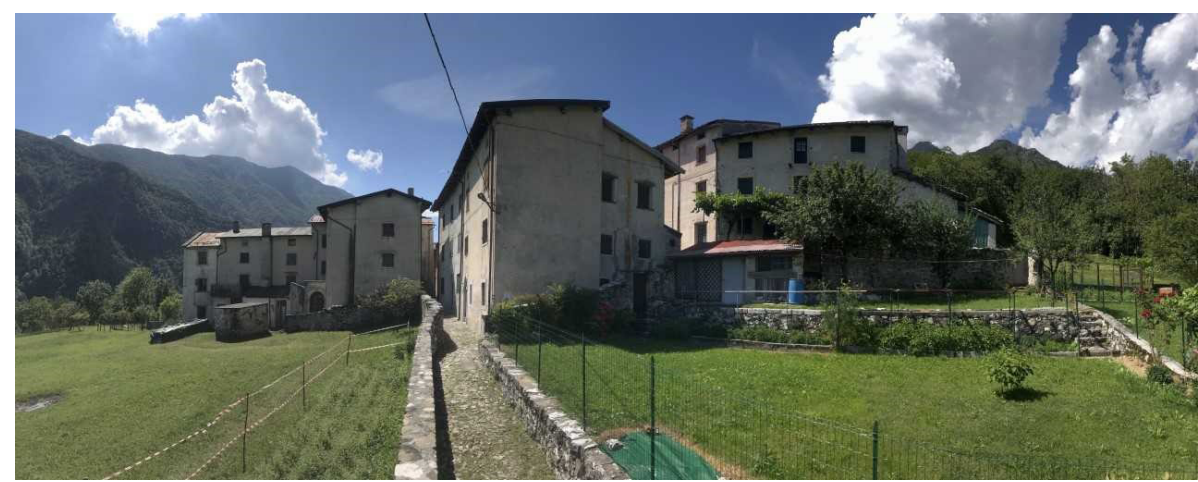

Abb. 3. Stavoli (Comune Moggio Udinese) - im Jahr 2005 noch ein echtes Geisterdorf mit weitgehend unbewohnbarem Baubestand. Bemerkenswerte Renovierungen in dieser abgelegenen Siedlung, in der 1951 noch 132 Bewohner lebten, weisen darauf hin, dass sie in der Gegenwart als Wohnsitz multilokal wohnender Zuwanderer neu entdeckt wird (eigene Aufnahme 2018).

analysieren, bevor vergleichbare Prozesse in den umliegenden Siedlungen darzulegen sind.

\subsection{Revitalisierung durch Zuzug in Dordolla}

Der grösste Ort im Val Aupa Dordolla zeigt mit dem dazugehörigen Nachbarweiler Drentus, wo sich eine fünfköpfige Bauernfamilie als ausgesprochene Pioniere erweist, den deutlichsten Aufschwung von existenzbedrohender Degradation - einhergehend mit einem Bevölkerungsverlust von $90 \%$ in der zweiten Hälfte des vorigen Jahrhunderts - zum auffallenden Einwohnerwachstum. Im Jahr 2018 hielten sich in Dordolla und Drentus neue Zuwanderer plus Remigranten und die autochthone Bevölkerung die Waage. Zusätzlich sind, vorzugsweise in den Sommermonaten, über 20 multilokale Bewohner monatelang ständig anwesend und in dieser Zeit hinsichtlich ihrer Aktivitäten von den ganzjährigen Einwohnern kaum zu unterscheiden. Abbildung 4 stellt die Bewohner von Dordolla und Drentus nach ihrer Herkunft unter Bezugnahme auf ganzjährig bewohnte Hausstätten und Zweitwohnsitze - im Sommer 2018 dar.

Aus Interviews geht klar hervor, dass die neuen Zuwanderer hauptverantwortlich für die Nutzung und Ausweitung der verbliebenen Kulturlandschaftsflächen, die Restaurierung des Baubestandes, den Wiederaufbau funktional und optisch wertvoller Trockensteinmauern, die Initialisierung eines spezifischen, saisonübergreifenden Fremdenverkehrs sowie für einen dynamischen Anstieg des Bekanntheitsgrades von Dordolla innerhalb und ausserhalb der Grenzen Friauls waren. Der zuletzt genannte Punkt bildet wiederum die Basis für den regen Tagestourismus, der neue und bestehende Strukturen stabilisiert. Die Wiedernutzung und Inwertsetzung alter Verkehrswege wird von den Zuwanderern und der autochthonen Bevölkerung gemeinsam betrieben.

Zudem erfordern die ungünstigen Besitzverhältnisse (Realteilung) mit ihrer überaus kleinteiligen Fragmentierung ein gemeinsames Handeln, da ein starres Festhalten an bestehenden Besitzverhältnissen Innovationen im Agrarsektor er-

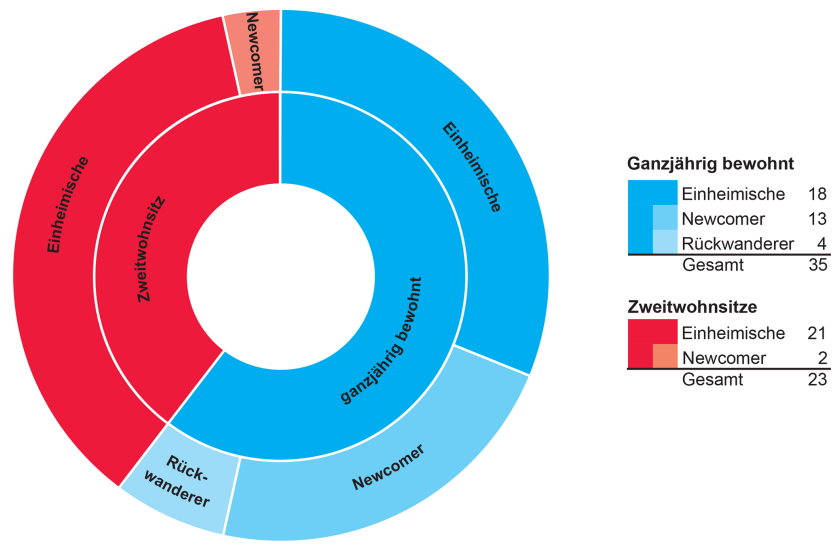

Abb. 4. Wohnstatus in Dordolla und Drentus 2018. Die beiden Ringe stellen den Wohnstatus der anwesenden Bewohner zum Zeitpunkt der Recherche dar (eigene Erhebungen).

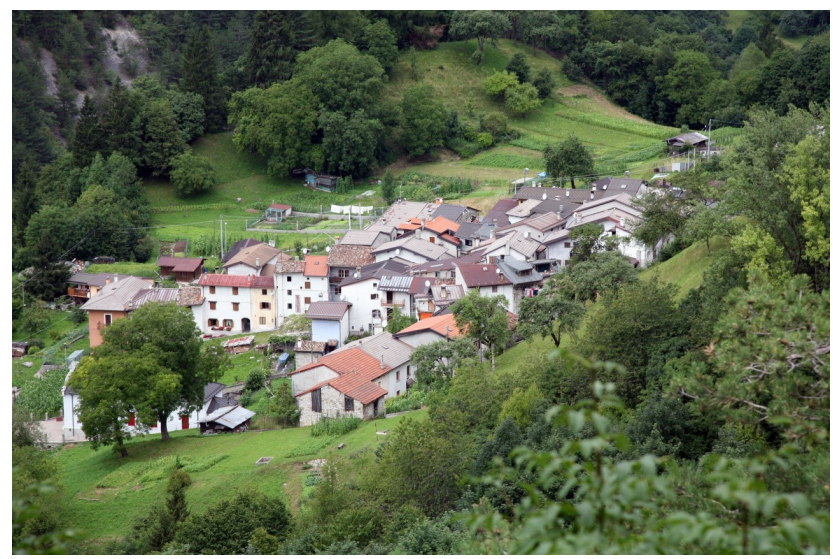

Abb. 5. Dordolla - von der partiellen Wüstung in den 1990er Jahren zum Zentrum der Innovationen durch neue Pioniere im friulanischen Canale di Ferro (eigene Aufnahme 2018). 
schweren würde. So gelang es der vorhin erwähnten Bauernfamilie aus Drentus - begünstigt durch lokale Verwandtschaftsverhältnisse der Bäuerin - über 3 ha Landbesitz und Nutzungsrechte zu vereinen, sodass landwirtschaftliche Tätigkeiten möglich sind.

\subsubsection{Agrarwirtschaft und Kulturlandschaft}

Die landwirtschaftlichen Aktivitäten dieser zugewanderten Familie konzentrieren sich vor allem auf die Nutzung von Agrarflächen und somit auf den Erhalt des Dauersiedlungsraumes. Dieser Aufwand rentiert sich nur durch die Symbiose mit sanftem Tourismus, der wiederum auf die in der Kulturlandschaft sichtbaren Intensivierungen zurückzuführen ist. Zudem gründete die Familie gemeinsam mit zwei Partnern aus der Gemeinde eine Kooperative zur Schafhaltung, -schlachtung und Fleischverarbeitung, wozu EUFördermittel lukriert wurden. Die primäre Funktion der neuen Schafherde ist jedoch das Offenhalten der verbliebenen Grünlandflächen, wodurch weitere Verwahrlosung und Verbuschung der Kulturlandschaft verhindert werden.

Die raumrelevante Wirkung dieser Landwirte (Produktion von Lamm- und Schweinefleisch, Käse und Schafwolle, Anbau von Kartoffeln, Hülsenfrüchten, Getreide und Gemüse für den Eigenbedarf sowie für Gäste und Touristen - Slow food) als Initiatoren der Revitalisierung Dordollas erstreckt sich indirekt auch auf weitere, entwicklungsfördernde $\mathrm{Zu}$ wanderung. Nicht zuletzt aufgrund der Vorbildwirkung und Pionierleistung siedelten sich in jüngster Vergangenheit weitere New Farmer an. Aktuelle Landnutzungskartierungen im Val Aupa zeigen, dass noch genügend Potential zur Intensivierung der landwirtschaftlichen Tätigkeiten vorhanden ist. Insgesamt sind die von den New Farmern wiedergenutzten Agrarflächen ein wesentlicher Bestandteil der Kulturlandschaftspflege (Beismann und Steinicke, 2019). Im Zusammenhang damit ist auch die Instandsetzung alter und Errichtung neuer Trockensteinmauern zu erwähnen, die vor zehn Jahren von einem neuen Zuwanderer begonnen wurden, mittlerweile abgeschlossen sind und in agrokulturellem Kontext häufig als Vorzeigeobjekt Beachtung finden.

\subsubsection{Gastronomie und Tourismus}

Die Bar Alimentari da Fabio, die auch Mittag- und Abendessen anbietet, spielt laut Interviewergebnissen eine zentrale Rolle für den Erhalt von Dordolla als Dauersiedlung: Dieser Gastronomiebetrieb ist für Einheimische, Zugewanderte und auswärtige Besucher das Kommunikationszentrum. Die Beherbergung von Gästen und Touristen erfolgt, wiederum von Zuwanderern betrieben, in Selbstversorgerhäusern in Dordolla sowie im Agriturismo Tiere Viere in Drentus. Darüber hinaus werden Führungen angeboten, zumal der aus Kärnten zugewanderte Bauer diplomierter Landwirtschaftsökologe ist und mittlerweile hohe Lokal- und Regionalkompetenz besitzt. Aus touristischer Perspektive hat die von der Bauern- familie gepachtete Alm über dem Ursprung der Aupa ausserdem hohes Entwicklungspotential.

Die Nutzung eines verfallenden Leerstandes als B \& B durch einen weiteren neuen Zuwanderer scheiterte während der Umbautätigkeit an bürokratischen Hürden; die Installation dreier Aussichtsplattformen in Dordolla inkl. Schautafeln konnte jedoch realisiert werden. Der neue, sanfte Tourismus, an dessen Etablierung sich auffallend viele Akademiker beteiligten, gehört zu den vielversprechenden neu geschaffenen Potentialen zur Stabilisierung und Ausweitung des Aufschwungs.

\subsubsection{Kunst und Kultur}

Bereits die ersten Zuwanderer, denen New Farmer und weitere neue Gebirgsbewohner - darunter ein britisches Autorenund Künstlerpaar - folgten, bewirkten mit Aktiven aus der lokalen Bevölkerung überregionale Impulse im künstlerischkulturellen Bereich.

Als Verein initiieren die neuen Bewohner gemeinsam mit Einheimischen neben Dorffesten mit landwirtschaftlichtraditionellen Schwerpunkten mehrtägige Kunstfestivals auch mit internationalen Künstlern. Dabei stellt die ansässige Bevölkerung oft ihre Netzwerke zur Verfügung und übernimmt weitgehend die Organisation. Diese Aktivitäten bewirken nicht nur eine während der Veranstaltungen starke Zunahme von Besuchern, sondern auch steigende mediale Präsenz des Ortes über Friaul hinaus (vgl. z. Bsp. Messaggero Veneto v. 28. September 2018, 14. Dezember 2018 und 24. September 2021).

Mehrfacher, auch längerer Arbeitsaufenthalt von Wissenschaftlern und Freiberuflern, von akademischen Exkursionsgruppen, Journalisten und Fernsehteams, arrivierten Künstlergruppen sowie die Filmproduktion von The New Wild, welche das Aupatal in Norditalien bekannt gemacht hat, verleihen dem Dorf den Flair eines intellektuellen Zentrums der Montagna Friulana. Dies scheinen nicht nur die Besucher, sondern auch die jungen Dordollesi zu schätzen, für die laut Befragungen die Abwanderung nicht mehr die einzige Option ist.

Seit mehr als einem Jahrzehnt finden sowohl wiederkehrende als auch spontane Kunst- und Kulturaktivitäten (Sagras, jährliches 10-tägiges Harvest-Festival, Cantiere Continuo oder Wanderfestivals) statt, die auch zur nichtagrarischen Inwertsetzung von Flurbrachen und zu künstlerischen Renovierungen des Baubestandes führen. Eine Intensivierung bzw. höhere Frequenz von Kulturveranstaltungen ist den Interviewergebnissen zufolge nicht erwünscht; offensichtlich ist bereits eine Sättigung eingetreten. Möglicherweise ist die Präsenz episodisch anwesender Persönlichkeiten aus Kunst, Wissenschaft und Kreativwirtschaft der wichtigste, wenn auch am schwersten quantifizierbare Ausdruck des Aufschwungs.

Die neuen Zuwanderer fungierten in Kooperation mit der lokalen Bevölkerung als Pioniere für alle zuvor genannten 
landwirtschaftlichen, touristischen und kulturellen Aktivitäten, mit denen vor Ort auch neue Arbeitsplätze verbunden sind. Bislang ist das „System Dordolla“ - abgesehen von einzelnen EU-Förderungen im künstlerisch-kulturellen Bereich - keinem von aussen gesteuerten Planungsprozess unterlegen.

\subsection{Klassifizierung revitalisierter peripherer Siedlungen}

In räumlicher Nähe zu Dordolla befinden sich Dörfer und Weiler, in denen um die Jahrtausendwende die völlige Entsiedelung fast schon greifbar war. Manche entsprechen dem Klischee der borghi più belli d'Italia, manche zeigen verfallene Ortszentren, die sowohl alte, passive Bewohner als auch kreative, multilokale Zuwanderer aufweisen; die meisten gleichen aktuell aber einem Labor für Revitalisierungsprozesse.

Von den untersuchten Siedlungen rund um Dordolla verzeichnen bis auf jene in der unmittelbaren Umgebung des Gemeindehauptortes Moggio Udinese (Chiandra und Pradis) alle übrigen unterschiedliche Zuwanderungsintensitäten, mindestens jedoch erstarkte, neue und zeitweise Frequentierung sowie eine Trendumkehr Richtung Revitalisierung. Tabelle 2 bietet dafür eine Übersicht.

Aus der Tabelle lassen sich drei Kategorien von Siedlungen feststellen:

- Orte ohne besondere Entwicklung: Die zwei bereits genannten Dörfer Pradis und Chiandra im unteren Abschnitt des Val Aupa, gleich ausserhalb des Gemeindehauptortes, waren zu keiner Zeit wüstungsgefährdet und sind hinsichtlich ihrer Erreichbarkeit diesem fast gleichzusetzen. Sie zeichnen sich durch stetige Bevölkerungsverluste ohne Gegentrend aus. Beide scheinen für neue Zuwanderer ebenso uninteressant zu sein wie die direkt im Canale di Ferro liegenden, nicht in die Erhebung einbezogenen Dörfer Ovedasso und Roveredo; gleichzeitig sind keine Prozesse innovativer oder zukunftstauglicher Entwicklung zu beobachten.

- Ehemalige Wüstungen ohne nennenswerte Revitalisierung: Im Untersuchungsgebiet ist hier nur der Doppelort Riulade di sotto und Riulade di sopra im Val Alba zu nennen. Diese Siedlungen sind keine echten Wüstungen mehr, da in beiden jeweils eine Person zeitweise lebt. Ausserdem kommen sporadisch weitere ehemalige Bewohner, um kleinere Renovierungsarbeiten zu leisten, damit der Baubestand nicht weiter verfällt.

- Ehemalige Wüstungen oder wüstungsgefährdete Orte mit nennenswerter Entwicklung: Alle übrigen in der Tabelle genannten Siedlungen gehören zu diesem Typus. Dabei finden sich die unterschiedlichsten Entwicklungsstadien: von Dörfern wie Dordolla und Grauzaria mit ständigen Bewohnern, auffallend gepflegter Kulturlandschaft und Viehhaltung (Rinder, Zie-

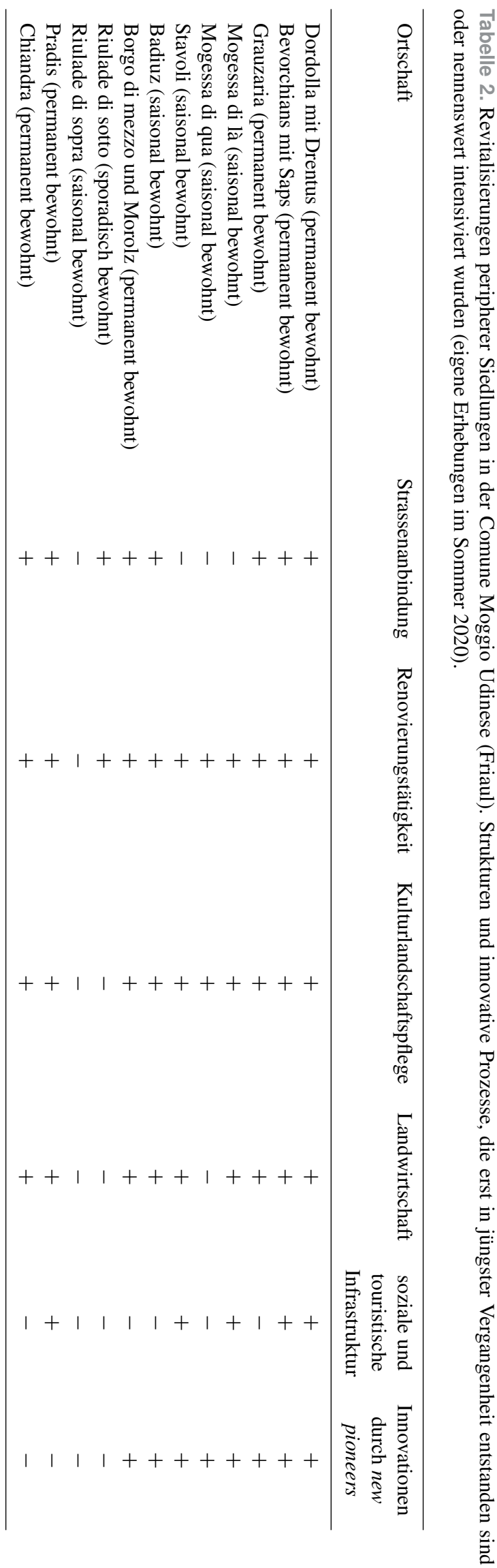

https://doi.org/10.5194/gh-77-71-2022 
gen und Schafe) sowie beachtlicher landwirtschaftlicher Tätigkeit und Tourismus über saisonal bewohnte Siedlungen, wo ebenso soziale Infrastruktur (Vereine, Gemeinschaftseinrichtungen) vorhanden ist und die Wiesen gemäht werden, bis hin zu ehemals verlassenen Orten. Diese sind zum Teil nur mit allradgetriebenen Fahrzeugen erreichbar, dennoch werden dort die Renovierung des Baubestandes von saisonal und episodisch anwesenden Personen durchgeführt und im Sommer zudem einfache gastronomische Dienstleistungen angeboten. Allen Siedlungen ist jedoch gemeinsam, dass Pioniere im Hinblick auf die Trendumkehr und neue Entwicklung vor Ort aktiv tätig sind.

\section{Konklusion}

Es wurde aufgezeigt, dass das Phänomen der neuen Gebirgsbewohner mittlerweile auch die Montagna Friulana im Osten der italienischen Alpen erfasst hat. Dadurch kam es zum allmählichen Abklingen der dort generationenlang vorherrschenden Entvölkerung. Besonders deutlich machen sich die Auswirkungen dieser neuen Zuwanderung in den ehemaligen Ghosttowns und wüstungsgefährdeten Siedlungen Friauls bemerkbar, und zwar nicht nur in demographischer, sondern insbesondere auch in sozio-ökonomischer, kultureller und kulturlandschaftlicher Hinsicht.

Die Forschungsergebnisse bestätigen die eingangs formulierten Annahmen. Wie sehr neue Zuwanderer, Rückwanderer sowie nur saisonal anwesende Bewohner Initiatoren und Träger der Revitalisierung alpiner Ortschaften sind, lässt sich im nordfriulanischen Canale di Ferro und hier wiederum am Modellfall Dordolla, aber auch in den übrigen Siedlungen im Hinterland der Gemeinde Moggio Udinese veranschaulichen. Die Frage, ob es in Friaul noch aktuell sterbende Dörfer oder Ghosttowns gibt, kann eindeutig verneint werden. Das Gegenteil ist der Fall. Welchen Stellenwert und welche Resilienz diese Ortschaften erreichen werden, wird sich jedoch erst in einigen Jahren zeigen. Die Coronakrise, welche die multilokale Bevölkerung offensichtlich dazu bewegt, ihre Wohnsitze in der Peripherie früher und länger aufzusuchen (Health Migration), kann beschleunigend wirken. Aktuell wird bereits deutlich, dass sich der Zuzug ins Gebirge in den letzten beiden Jahrzehnten verstärkt hat - sowohl in entlegenen Siedlungen als auch in leichter erreichbaren. Nachteile fehlender Infrastruktur und Grundversorgung werden teilweise nicht mehr als Hinderungsgrund wahrgenommen oder kreativ ausgeglichen.

Unter Bezugnahme auf die Kulturlandschaft nehmen New Farmer mit Viehhaltung die tragende Rolle ein, gefolgt von Remigranten und alteingesessenen Bauern. Das Potential der neuen und gleichzeitig traditionellen Landwirtschaft darf trotz kleiner, fragmentierter Nutzflächen nicht unterschätzt werden, indem Vergleiche zur konventionellen Landwirtschaft oder zum globalisierten Markt vorgenommen werden.
Der unmittelbare regionale Wert dieser agrarischen Tätigkeiten als Katalysator für lokale Wirtschaftskreisläufe, als Anziehungspunkt für sanften Tourismus, oftmals auch als letzte Chance für die Weitergabe eines tradierten, singulären Wissens über alte Obst- und Getreidesorten sowie als Voraussetzung für den Erhalt oder die Wiedernutzung der Kulturlandschaft und nicht zuletzt als Vorbildwirkung ist evident. Bislang steht fest, dass die verschiedenen innovativen Landwirte ein Aspekt eint: Nirgendwo handelt es sich um eine konventionelle Hofübernahme, sondern um New Farming als neuer gesellschaftsrelevanter Faktor.

Auch in anderen Aktionsfeldern sind Aktivitäten der Neuzuwanderer relevant: Akteure aus Kreativwirtschaft, Kunst, Kultur, Bildung und Forschung wirken sich positiv auf die Revitalisierung der Siedlungen aus. Wie die Filmproduktion The New Wild zeigt, erlangte vor allem Dordolla als das Innovationszentrum alpiner Periphergebiete in Friaul überregionale Bekanntheit und Aufmerksamkeit. Im Bereich Gastronomie und Tourismus ging von den Pionieren - neben umwelt- und landschaftsdidaktischen Projekten zur Inszenierung und touristischen Inwertsetzung erfolgreicher Revitalisierungsmassnahmen - die Vermietung mehrerer Selbstversorgerhäuser im Sinne des Albergo Diffuso-Gedankens aus, die im Sommer 2020 und 2021 gerade wegen der CoronaKrise stark nachgefragt waren.

Im Untersuchungsgebiet konnten, im Gegensatz zu einzelnen innovationsblockierenden Einheimischen, keine entwicklungshemmenden multilokalen Einwohner nachgewiesen werden. Die permanent anwesenden Newcomer sind in der Regel gut in die Dorfgemeinschaft integriert. Allerdings gibt es Pioniere in Streusiedlungen (case sparse), die sich dort individuell verwirklichen. Durch die jahrzehntelange Abwanderung mit daraus resultierenden Verfallserscheinungen in der Kulturlandschaft sieht nicht nur die einheimische Bevölkerung der Montagna friulana in den Neuzuwanderungen Chancen für eine Trendumkehr und steht diesen insgesamt nicht kritisch, sondern - von wenigen Ausnahmen abgesehen - ausgesprochen wohlwollend gegenüber.

Die Forschungsergebnisse belegen insbesondere, dass neue ökonomische und kulturlandschaftliche Prozesse lediglich von wenigen Neuzuwanderern ausgehen. Oft genügt dafür die Leistung eines einzigen Akteurs. Auf keinen Fall gilt im Untersuchungsgebiet, dass die Anzahl der neuen $\mathrm{Zu}$ wanderer für Revitalisierung und Entwicklung entscheidend ist! Vielmehr resultieren diese aus Innovation und Identifikation mit dem Zielort und der Dorfgemeinschaft. Es liegt daher nahe, auch in weiterführenden Studien nicht länger auf unterschiedliche zeitliche Muster des Aufenthalts oder gar auf den Meldestatus zu fokussieren, sondern auf lokale Handlungs- und Wirkungsmuster sowie, bei multilokalen Bewohnern, auf die Beziehung zwischen ihren Wohnorten und deren Bedeutung.

Eindeutig entbehren sämtliche untersuchten Intensivierungsprozesse einer lokal oder regional gesteuerten Planung bzw. einer zielgerichteten Entwicklungsstrategie. Vielmehr 
werden sie in der Regel als individuelle Projekte von multilokalen Zuwanderern initiiert und getragen. Vor allem dann, wenn diese Pioniere mit der lokalen Bevölkerung kooperieren, lässt sich ein hoher Grad an Revitalisierung, möglicherweise sogar Kontinuität als Dauersiedlungsraum erreichen. Der Modelfall Dordolla zeigt somit die Verbindung endogener und exogener Einflussfaktoren und kann daher auch zu Strategiediskussionen in der Regionalentwicklung beitragen.

Die Revitalisierungsprozesse und Leistungen der neuen Pioniere lassen sich aus den Ergebnissen der Feldforschung resultierend auf die ehemaligen Geisterdörfer und partiellen Wüstungen in der gesamten Montagna Friulana übertragen. Demnach handelt es sich im Untersuchungsgebiet nicht um ein singuläres Phänomen: Da dort ausser Abwanderung und Degradierung keine weiteren lokal- und regionalspezifischen Merkmale - weder extreme Peripherie noch hervorstechende landschaftliche Besonderheiten bzw. Attraktivitäten - vorliegen, sind potentielle Wiederbesiedlung und Revitalisierung der Kulturlandschaft in anderen demographischen Problemräumen des Alpenbogens, insbesondere mit Fokus auf Prozesse und Strukturen der Amenity Migration, nicht unrealistisch.

Um konkrete Strategien und Konzepte aus den untersuchten Pionierleistungen abzuleiten, wäre auf Begriffe wie Postproduktivismus, Kreislaufwirtschaft, Bottom-upRegionalentwicklung und synergie- sowie symbioseorientiertes Handeln zu fokussieren. Die Motivationen der neuen Pioniere zugrunde legen hiesse zudem, eine Diskussion über Gegenentwürfe zum System globalisierter Ballungsräume zu führen, die in eine Neubewertung von Stadt-LandBeziehungen münden könnte.

Datenverfügbarkeit. Die Datensätze für diesen Artikel können aus Datenschutzgründen nicht veröffentlicht werden.

Autorenmitwirkung. MB ist für Kap. 6 und 7, PČ für Kap. 3 und 5, ES für Kap. 1, 2 und 4 verantwortlich.

Interessenkonflikt. Die Autor*innen erklären, dass kein Interessenkonflikt besteht.

Haftungsausschluss. Anmerkung des Verlags: Copernicus Publications bleibt in Bezug auf gerichtliche Ansprüche in veröffentlichten Karten und institutionellen Zugehörigkeiten neutral.

Begutachtung. This paper was edited by Benedikt Korf and reviewed by three anonymous referees.

\section{Literatur}

Ayob, N., Teasdale, S., and Fagan, K.: How social innovation ,Came to Be': Tracing the evolution of a contested concept, J. Social Policy, 45, 635-653, https://doi.org/10.1017/S004727941600009X, 2016.

Beismann, M. and Steinicke, E.: New In-migrants - New Chances: The Revitalization of Abandoned Villages from Outside, in: Alpine Refugees. Immigration at the Core of Europe, edited by: Perlik, M., Galera, G., Machold, I., and Membretti, A., Cambridge, UK, 201-211, ISBN 1-5275-3672-6,, 2019.

Beismann, M., Löffler, R., Walder, J., Warmuth, W., and Steinicke, E.: Newcomers in the Alps: Benefits of having ,,Second Homers". A Case Study in the Eastern Italian Alps/Nuovi abitanti nelle Alpi: I vantaggi di avere proprietary di seconde case. Un caso di studio nelle Alpi orientali, Mountain Dossier, Scientific Review of Dislivelli.Eu, 4, Housing Policies in Mountain Areas, ISBN 2039-5442, 2015.

Bender, O. and Kanitscheider, S.: New Immigration into the European Alps: Emerging Research Issues, Mount. Res. Dev., 32, 235-241, 2012.

Berry, B. J. L. (Hrsg.): Urbanization and Counterurbanization, Beverly Hills, Sage Publications, 1976.

Čede, P. und Steinicke, E.: Ghost towns in den Ostalpen: das Phänomen der Entvölkerung im friulanischen Berggebiet (Italien), Geogr. Helv., 62, 93-103, https://doi.org/10.5194/gh-62-932007, 2007.

Čede, P., Deissl, G., Löffler, R., and Steinicke, E.: The Eastern Austrian Alps - their Exceptional Demographic Status in the Alpine Region, Eur. Countryside, 10, 634-651, https://doi.org/10.2478/euco-2018-0035, 2018.

Cretton, V.: In search of a better world in the Swiss Alps, Lifestyle migration, quality of life, gentrification, in: Utopia and Neoliberalism. Ethnographies of rural spaces, edited by: Horáková, V. H., Boscoboinik, A., and Smith, R., Berlin, 105-125, ISBN 9783-643-80215-6, 2019.

Dematteis, M., Di Gioia, A., and Membretti, A.: Montanari per forza, Franco Angeli Editore, Milano, online aufrufbar: http: //goo.gl/j7Qbqg (letzter Zugriff: 21. Januar 2022), 2018.

Dissart, J. C. and Marcouiller, D. W.: Rural Tourism Production and the Experience-Scape, Tourism Anal., 17, 691-704, 2012.

Duchêne-Lacroix, C., Hilti, N., und Schad, H.: Das Projekt „,Multilokales Wohnen in der Schweiz", in: Kompendium Multilokale Lebensführung und räumliche Entwicklungen, Herausgeber: Arbeitskreis Multilokale Lebensführung und räumliche Entwicklungen der Akademie für Raumforschung und Landesplanung, Akademie für Raumforschung und Landesplanung, Hannover, 23, online aufrufbar: http://shop.arl-net. de/multilokale-lebensfuehrung.html (letzter Zugriff: 23. Oktober 2021), 2018.

Fassio, G., Battaglini, L. M., Porcellana, V., and Viazzo, P. P.: The Role of the Family in Mountain Pastoralism - Change and Continuity, Mount. Res. Dev., 34, 336-343, 2014.

Fourny, M.-C. (Ed.): Montagnes en mouvements. Dynamiques territoriales et innovation social, PUG et UGA éditions, Grenoble, 2018.

Garcia, F.: Pouvoirs en souffrance: neo-ruraux et collectivités rurales du Pays de Sault Oriental, Études Rur., 65, 101-108, 1977. 
Gosnell, H. and Abrams, J.: Amenity migration: diverse conceptualizations of drivers, socioeconomic dimensions, and emerging challenges, Geo J., 764, 303-322, 2011.

Graf, F.: Migration in den Alpen. Handlungsspielräume und Perspektiven, Kultur und soziale Praxis, gefördert durch: Institut für Kulturforschung Graubünden und Swiss National Science Foundation (SNSF), https://doi.org/10.14361/9783839455647, 2020.

Graves, P. E.: Migration and climate, J. Reg. Sci., 20, 227-237, 1980.

Gretter, A., dalla Torre, C., Maino, F., and Omizzolo, A.: „New Farming" as an example of Social innovation responding to challenges of inner mountain areas of Italian Alps, Rev. de Géogr. Alp., 107, 2, https://doi.org/10.4000/rga.6106, 2019.

Grüner, B. und Konzett, S.: Gegen den Strom - Neue Zuwanderung in den alpinen Westen Österreichs, Innsbr. Jahresber. 2016-2017, 121-138, online aufrufbar: https://www.uibk.ac.at/geographie/ igg/berichte/2017/pdf/8_gruener_konzett.pdf (letzter Zugrif: 3. Oktober 2021), 2017.

Hägerstrand, T.: Innovation Diffusion as a Spatial Process (transl. by A. Pred), Geogr. Rev., 59, 309-311, 1969.

ISTAT - Istituto Nazionale di Statistica: Popolazione e movimento anagrafico dei comuni (Statistiche demografiche/Annuario demografico), Roma, online aufrufbar: http://www.demo.istat.it (letzter Zugriff: 1. November 2021), $1951 \mathrm{ff}$.

Jacob, J. C.: New Pioneers, Pennsylvania Press, University Park, USA, ISBN 978-0-271-02989-4, 1997.

Kordel, S., Weidinger, T., and Jelen, I. (Eds.): Processes of Immigration in Rural Europe. The Status Quo, Implications and Development Strategies, Cambridge Scholars Publishing, Newcastle upon Tyne, UK, ISBN 1-5275-0676-2, 2018.

Löffler, R. and Steinicke, E.: Amenity Migration in the U.S. Sierra Nevada, Geogr. Rev., 97, 67-88, 2007.

Löffler, R., Walder, J., Beismann, M., Warmuth, W., and Steinicke, E.: Amenity Migration in the Alps: Applying Models of Motivations and Effects to 2 Case Studies in Italy, Mount. Res. Dev., 36, 484-493, 2016.

Mayer, H. and Meili, R.: New Highlander Entrepreneurs in the Swiss Alps, Mount. Res. Dev., 36, 267-275, https://doi.org/10.1659/MRD-JOURNAL-D-16-00040.1, 2016.

Mayring, P.: Qualitative Inhaltsanalyse - Abgrenzungen, Spielarten, Weiterentwicklungen, Forum Qualitative Sozialforschung/Forum: Qualitative Social Research, 20, 1-15, https://doi.org/10.17169/fqs-20.3.3343, 2019.

McIntyre, N.: Rethinking amenity migration: integrating mobility, lifestyle and social-ecological systems, Erde, 140, 229-250, 2009.

Mercier, C. and Simona, G.: Le néo-ruralisme. Nouvelles approches pour un phénomène nouveau, Rev. de Géogr. Alp., 71, 253-265, 1983.

Meyer, H.: Attraktive Kulturlandschaften als Grundlage ländlicher Entwicklung, in: Die Kulturlandschaft im Berggebiet Österreichs. Politiken zur Sicherung von Umwelt- und Kulturleistungen und ländliche Entwicklung, 43, Herausgeber: Hovorka, G., Forschungsberichte der Bundesanstalt für Bergbauernfragen, Wien, 161-185, online aufrufbar: https://j1dev.agrarforschung.at/jdownloads/Publikationen/ Archiv/BABF/Forschungsberichte/fb43.pdf (letzter Zugriff: 5. Mai 2021), 1998.
Moss, L. A. G.: The Amenity Migrants. Seeking and Sustaining Mountains and their Cultures, Wallingford, UK, Cambridge, USA, ISBN 978-0851990842, 2006.

Moss, L. A. G. and Glorioso, R. S.: Global Amenity Migration Transforming Rural Culture, Economy and Landscape, Kaslo, Canada, ISBN 978-0-9936351-0-6, 2014.

Perlik, M.: The Specifics of Amenity Migration in the European Alps, in: The Amenity Migrants - seeking and sustaining Mountains and their Cultures, edited by: Moss, L. A. G., Wallingford, UK, Cambridge, USA, 215-231, ISBN 978-0851990842, 2006.

Perlik, M.: Alpine gentrification: The mountain village as a metropolitan neighbourhood, Rev. de Géogr. Alp., 99, 1, https://doi.org/10.4000/rga.1370, 2011.

Perlik, M. and Membretti, A. A.: Migration by necessity and by force to Mountain Areas: An Opportunity for Social Innovation, Mount. Res. Dev., 38, 3, 250-264, https://doi.org/10.1659/MRDJOURNAL-D-17-00070.1, 2018.

Perlik, M., Galera, G., Machold, I., and Membretti, A. (Eds.): Alpine Refugees. Immigration at the Core of Europe, Cambridge, UK, ISBN 1-5275-3672-6, 2019.

Schnell, R., Hill, P. B., und Esser, E.: Methoden der empirischen Sozialforschung, München, ISBN 9783110577327, 2018.

Steinicke, E.: Friaul. Bevölkerung und Ethnizität, Innsbr. Geogr. Stud., 19, Innsbruck, Austria, ISBN 3-901182-19-5, 1991.

Steinicke, E. und Löffler, R.: „New Highlanders“ in den Alpen. Das Ende der Entvölkerung in den Periphergebieten?, Geogr. Rdsch., 71, 32-37, 2019.

Steinicke, E., Walder, J., Löffler, R., and Beismann, M.: Autochthonous Linguistic Minorities in the Italian Alps: New Legislation - New Identifications - New Demographic Processes, Rev. de Géogr. Alp. [En ligne], 99, 2, https://doi.org/10.4000/rga.1454, 2011.

Steinicke, E., Čede, P., and Löffler, R.: In-migration as a new process in demographic problem areas of the Alps. Ghost towns vs. amenity settlements in the alpine border area between Italy and Slovenia, Erdkunde, 66, 329-344, 2012.

Steinicke, E., Čede, P., Löffler, R., and Jelen, I.: Newcomers nelle regioni periferiche delle Alpi. Il caso dell' area di confine tra Italia e Slovenia nelle Alpi Giulie, Riv. Geogr. Ital., 121, 1-20, 2014.

Tschumi, P., Winiger, A., Wirth, S., Mayer, H., und Seidl I.: Wachstumsunabhängigkeit durch Soziale Innovationen? Eine Analyse potenzieller Wachstumswirkungen von Sozialen Innovationen im Schweizer Berggebiet, in: Postwachstumsgeographien. Raumbezüge diverser und alternativer Ökonomien, Herausgeber: Lange, B., Hülz, M., Schmid, B., und Schulz, C., transkript-Verlag, Bielefeld, 117-137, https://doi.org/10.14361/9783839451809-009, 2020.

Varotto, M. and Lodatti, L.: New Family Farmers for Abandoned Lands, Mount. Res. Dev., 34, 315-325, 2014.

Vejre, H., Abildtrup, J., Andersen, E., Andersen, P. S., Brandt, J., Busek, A., Dalgaard, T., Hasler, B., Huusom, H., Kristensen, L. S., Kristensen, S., and Praestholm, S.: Multifunctional agriculture and multifunctional landscapes - land use as interface, in: Multifunctional land use: Meeting future demands for landscape goods and service, edited by: Mander, Ü., Wiggering, H., and Helming, K., Heidelberg, Berlin, Germany, 93104, ISBN 978-3-540-36763-5, https://doi.org/10.1007/978-3540-36763-5_1, 2007. 
Viazzo, P. P. and Zanini, R. C.: Taking advantage of emptiness? Anthropolical perspectives onmountain repopulation and spaces of cultural creativity in Alpine areas, Rev. de Géogr. Alp., 102, 1-12, 2014.

Warmuth, W., Beismann, M., Walder, J., Löffler, R., und Steinicke, E.: Die Wiederbelebung der Alpendörfer - Ein Blick in den Westen, Innsbr. Geogr. Stud., 40, 437-452, 2016.

Wilson, G. A.: The spatiality of multifunctional agriculture: A human geography perspective, Geoforum, 40, 269-280, 2009.

Zäch, C., Schulz, T., Waltert, F., und Pütz, M.: Landscape and Amenity Migration: Die Rolle von Landschaft für Wanderungsbewegungen in den ländlichen Raum der Schweiz, WSL Berichte 22, Eidg. Forschungsanstalt für Wald, Schnee und Landschaft WSL, Birmensdorf, online aufrufbar: https://www.dora. lib4ri.ch/wsl/islandora/object/wsl:9083 (letzter Zugriff: 20. Oktober 2021) 2015.
Zanini, L.: Friuli migrante, Doretti editore, Udine, Italia, 1964.

Zimmerli, J.: Zweitwohnungsbesitzer und Wohnraumentwicklung in den Regionen Albula und Prättigau/Davos, online aufrufbar: https://www.gemeindedavos.ch/dl.php/de/61ba61ddd3e96/ 21-1206_Davos_-_Prasentation_Infoveranstaltung_FINAL.pdf, letzter Zugriff: 15. Dezember 2021. 